\title{
The Rice Impurity Vision Inspection Method based OpenCV
}

\author{
Ying $\mathrm{Hou}^{1, \mathrm{a}}$, Guicai Wang ${ }^{2, \mathrm{~b}}$, Zhengyi Cui ${ }^{2, \mathrm{c}}$ \\ ${ }^{1}$ Zhengzhou Normal University, ZhengZhou, 450044, China \\ ${ }^{2}$ HeNan University of Technology, ZhengZhou, 450001, China \\ ahouyin69@163.com, bwangguicai@tom.com, '1034372362@qq.com
}

Keywords: Rice impurity; Machine vision; Grain quality

\begin{abstract}
The paper proposed a rice impurity vision inspection method based OpenCV, which was using OpenCV software development tools to significantly enhance impurity information and showing details of impurity. Secondly the paper could detect rice impurity and then improve accuracy of vision inspection by OTSU image binary method. Finally region location and detection of rice impurity would were obtained according to parameters of area for connected domains in rice impurity images. The Blob algorithm could extract those connected domains. The experimental results shown that the proposed algorithm could accurately not only detect the number but also label the location of rice impurity, which has important significance to perfect rice impurity real-time inspection technology and realize grain safety.
\end{abstract}

\section{Introduction}

The impurity content was an important index to distinguish the rice quality, which has a direct impact on its market competitiveness. Rice impurity inspect was an effective means to ensure the rice quality[1]. At present, the traditional rice impurity manual inspection method was very time-consuming and inefficient. The new inspection method of rice quality included sampling voice recognition method, Near-Infrared Absorption method and vision inspection method[2]. Since the beginning of 1980s, foreign scholars began to apply vision technology in rice identification and classification research, which could open up a new way for rapid image quality inspect and classification[3]. Vision inspection method has the advantages of high accuracy, small amount of labor, image visualization, easy to integrate with existing inspection software systems of grain quality and so on, Vision inspection has become a research focus in grain quality inspection field, lots of researcher had analyses and studies and obtained gratifying achievement [4].

The scholars made wide range of research on rice impurity vision inspection method, while had achieved some fruitful results. Those mainly included: since 1983 Sendaobo had extensive research on rice Identification and classification, such as of rice varieties in whole grain, granulated, Leis, grain, grade, and recognition method of different type rice[3]. Liu inspected rice milling degree by digital image process technology, which greatly improved efficiency compared to traditional methods[5]. Yadav used image analysis method to inspect rice milling degree. Meanwhile the amount of rice was calculated through extracting morphology features of rice (such as length, perimeter, projected area and so on) in the static rice image[6]. Wan applied range estimation, neural network and hybrid classification methods to inspect rice quality. Which the result was good but the classification speed was slow[7]. Rahul presents an efficient method for calculating the size of Oryza sativa L rice using machine vision along with detection of chalky and broken rice with improved accuracy compared with human inspector[8].

Recently rice impurity vision inspection got some progress and researchers had proposed a variety of detection algorithms. However when using the above algorithms to apply in the actual rice impurity, There were some urgent problems to be solved: existing storage pests vision inspection methods were large complexity of time and low computational efficiency so that fail to meet the rice impurity online inspection requirements. In addition the detection stressed the number, integrity and other appearance index, the actual research of pure impurity inspection was still few[9]. Moreover OpenCV was 
released under a BSD license and hence it was free for both academic and commercial use. OpenCV was designed for computational efficiency and with a strong focus on real-time applications[10].In order to make rice impurity vision inspection method can be successfully applied in practice, the paper used OpenCV development tool to achieve automatic detection of rice impurity, so that could facilitate and reduce the workload of the relevant personnel.

\section{Algorithm Description}

In order to reduce the workload of manual rice impurities inspection and overcome the shortcomings of the existing vision inspection method, the paper proposed a rice impurity vision inspection method based OpenCV. The proposed algorithm flow was shown in Fig. 1. The concrete steps mainly included gray processing, image binarization and Blob detection.

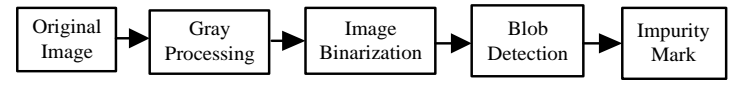

Figure.1 the processing steps of rice impurity vision inspection method

\section{Operating Environment}

Software Development Environment:

Visual Studio 2010, OpenCV -3.1.0;

Hardware Development Environment:

CPU: Intel(R) Core(TM) i5-3210M CPU @2.50GMz;

Operating system: Window 764 bit;

Memory: 4.0G;

Hard disk: $50 \mathrm{G}$

\section{Gray Processing}

The color image has large information. But sometimes the gray image could provide information to process and improve operation speed.

OpenCV code was as follows:

cvCvtColor(pSrcImage, pGrayImage, CV_BGR2GRAY);

The histogram expressed gray distribution and described the proportion of gray level in the image [11]. The histogram was indicated by 2D coordinate system, the concrete relationship as shown in the Eq. 1:

$$
P\left(\mathrm{r}_{k}\right)=n_{k} / N,
$$

Where $\mathrm{N}$ was the total number of pixels in the image; $n_{k}$ was the number of pixels for the K gray level; $r_{k}$ was the K gray level; $P\left(r_{k}\right)$ was the appeared probability of the K gray leve.

OpenCV code was as follows:

CvHistogram *pcvHistogram = cvCreateHist(1, \&nHistSize, CV_HIST_ARRAY, pfRanges); cvCalcHist(ppImage, pcvHistogram);

\section{Image Binarization}

The images usually contained noise, background and target. We could select the fixed threshold T and divided image into two parts. The pixels were greater than $\mathrm{T}$ and less than $\mathrm{T}$. That is the original image was divided into the background and the object. The specific implementation as shown Eq. 2. 


$$
f(x, y)=\left\{\begin{array}{ll}
1, & f(x, y) \geq T \\
0, & f(x, y)<T
\end{array},\right.
$$

The target object and background effectively not only distributed in the two gray scales. It required two or more than two threshold to obtain the target object.

$$
f^{\prime}(x, y)=\left\{\begin{array}{cc}
1, & \mathrm{~T}_{1} \leq f(x, y) \leq T_{2}, \\
0, & \text { other }
\end{array}\right.
$$

Due to the Otsu binarization algorithm with the convenience and immediacy, the paper adopted Otsu to binary process rice impurity image [12]. The essence Otsu method was an adaptive threshold method. According to gray value distribution features of the target image, this was divided into the background and objectives. For one rice impurity image $X$, according to between-cluster variance $\sigma$, targets and background could be divided and finally finished image binarization through the total average gray level of the image $\mu$, the $\mu_{0}$ average gray level of the target $C_{0}$ and it's the proportion of the image area $\mu_{0}, C_{1}$ the average gray level of the background and it's the proportion of the image area $\mu_{1}$.

OpenCV code was as follows:

cvThreshold(srcImage,biImage,255,255, CV_THRESH_OTSU | CV_THRESH_BINARY);

\section{Blob Detection}

The accurate detection of rice impurity was an effective method to ensure rice quality. The paper used Blob algorithm to analysis connected domain for the binary image of rice impurity and achieve the purpose of inspects impurity [13].

The paper used area parameter $S(\bullet)$ to measure size of target area. For the patch area $R(x, y)$, $S(\bullet)$ was defined as the number of pixels in this area:

$$
S(\mathrm{R} .(\mathrm{x}, \mathrm{y}))=\sum f(x, y),(x, y) \in R_{\bullet}(x, y) \text {, }
$$

Where $(x, y)$ was coordinate value of pixel; $f(x, y)$ was the gray value of pixel (black equal to 0 , white equal to 1$)$.

OpenCV code was as follows:

for(; pcvSeq != 0; pcvSeq = pcvSeq->h_next)

\{

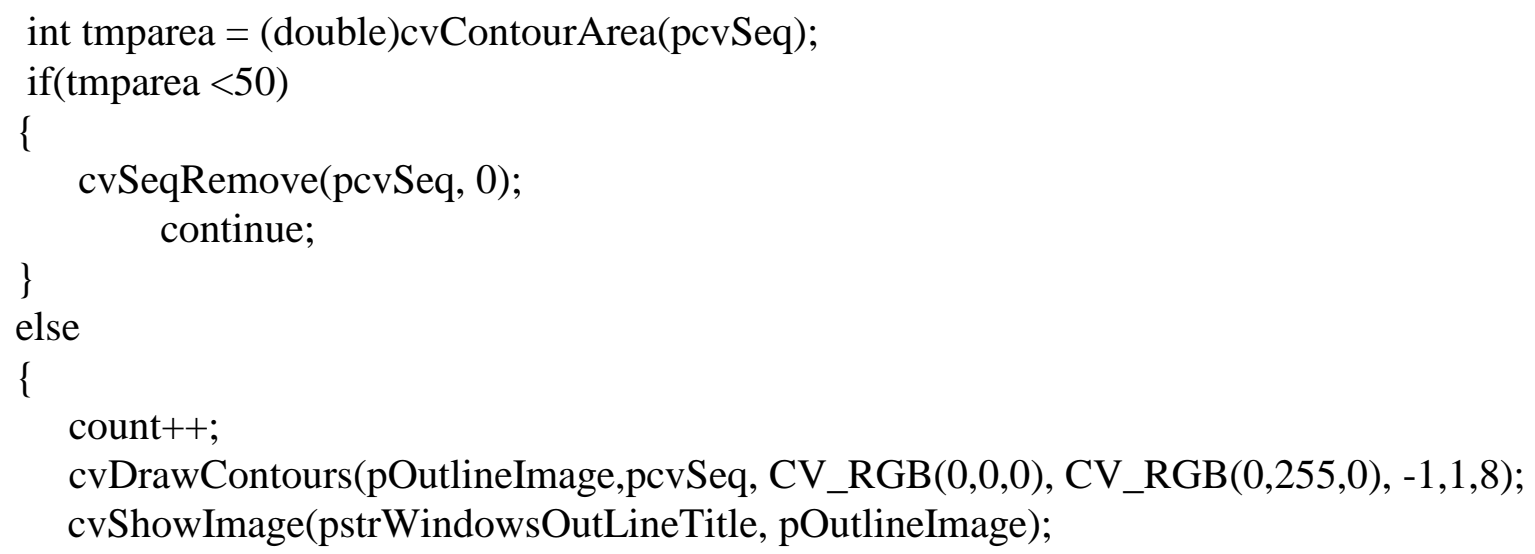

The paper proposed vision inspection method could fast, accurate and non-destructive automatic inspect rice impurity by area parameter $S(\bullet)$. 


\section{Analysis of Experimental Results}

The experimental rice images been taken by vision device. The images resolution was set to $640 \mathrm{x}$ 853. Due to space limitations, the paper only gave the two sets of rice impurity images (as shown in Fig. 2) to evaluate the proposed algorithm. A group of most of the impurities volume and area is less than the volume and area of the rice; another group of impurity volume and area for the volume and area of rice.
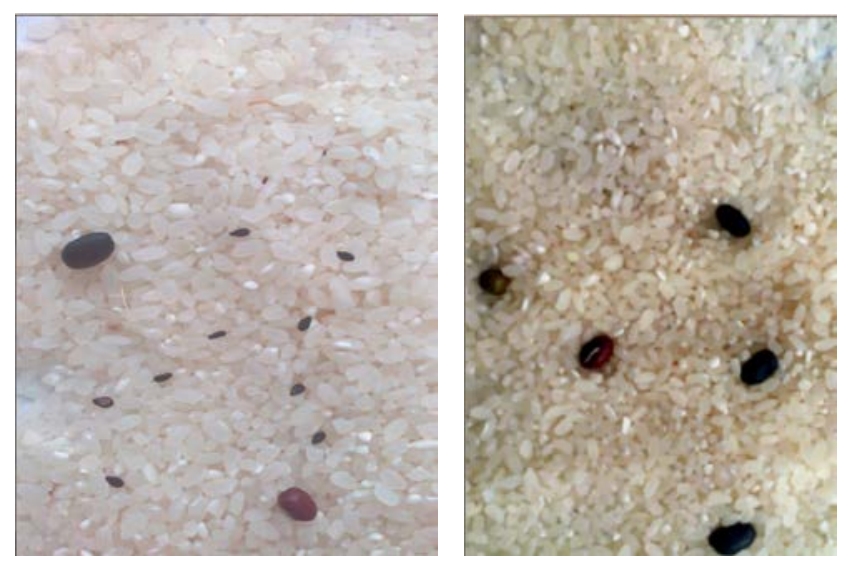

Figure. 2 the rice impurity images

It was not difficult to see from Fig. 3 that the gray images of rice impurity could help to highlight the impurity contour, weak the impurity details and realize the goal of enhancing the rice impurity image.
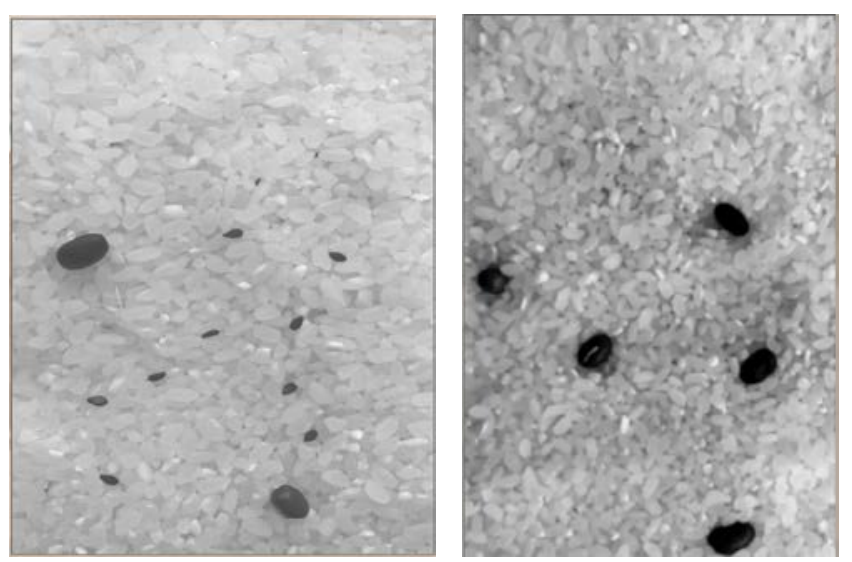

Figure.3 the gray images of rice impurity

It was easy to see that the gray level distribution of the rice impurity image from Fig. 4, the gray level of rice occupied area was the largest. Meanwhile the gray level of impurity occupied area was the least. This situation was very suitable for the binarization method of adaptive threshold.

The experiment adopted Otsu method binary process detected impurity from containing background and noise rice image. This could show details of binary image and had contributed to improve the inspection accuracy of impurity. The image binarization results shown in Fig. 5.

In the experiments the paper used blob algorithm to quickly and accurately extract connected regions of the rice impurity image, and calculated out area and perimeter parameters of connected domain according to the chain code list and linear list. Finally the paper could locate the rice impurity. This part was very important for the whole rice impurity inspection. The contours result of rice impurity was obtained by the Blob algorithm as shown in Fig. 6. 

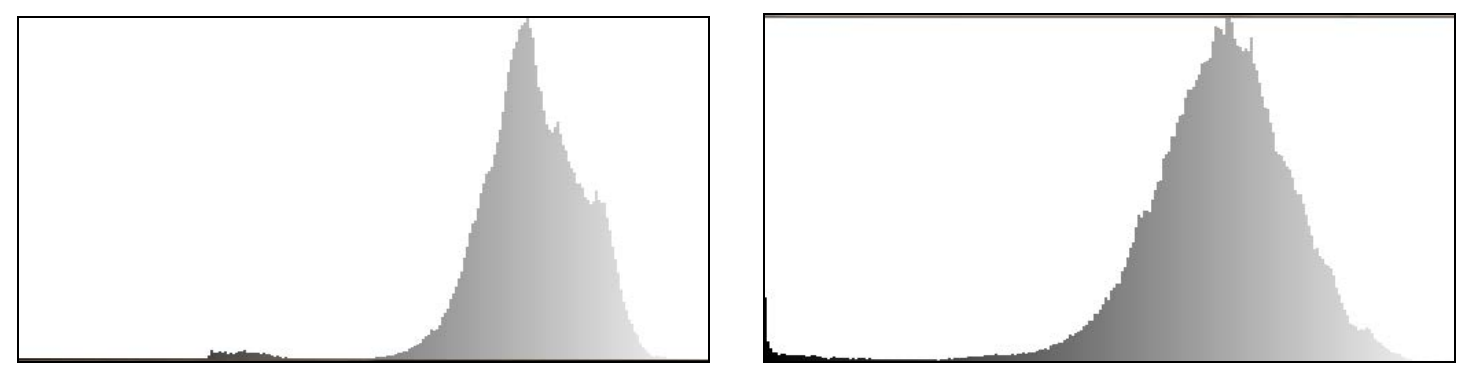

Figure.4 the gray histogram of rice impurity
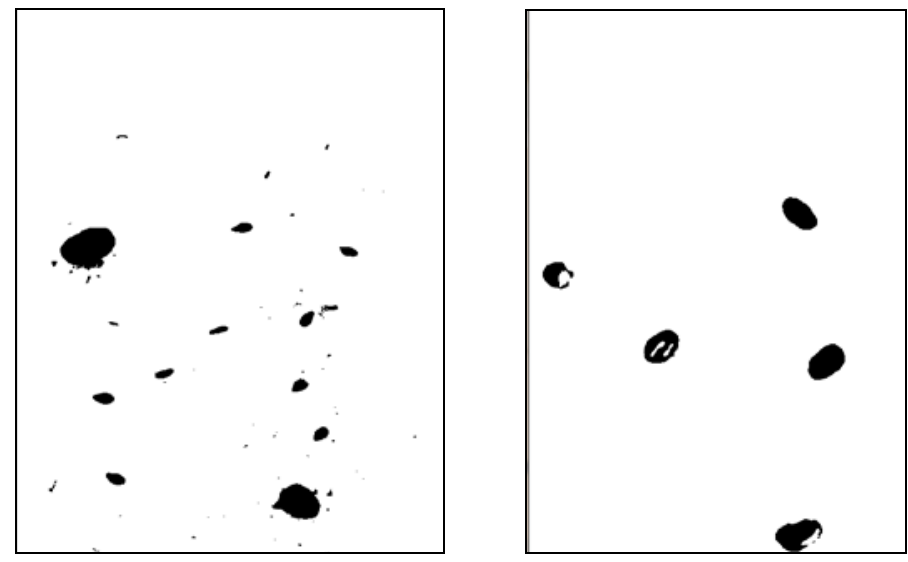

Figure.5 the binary images of rice impurity using Otsu
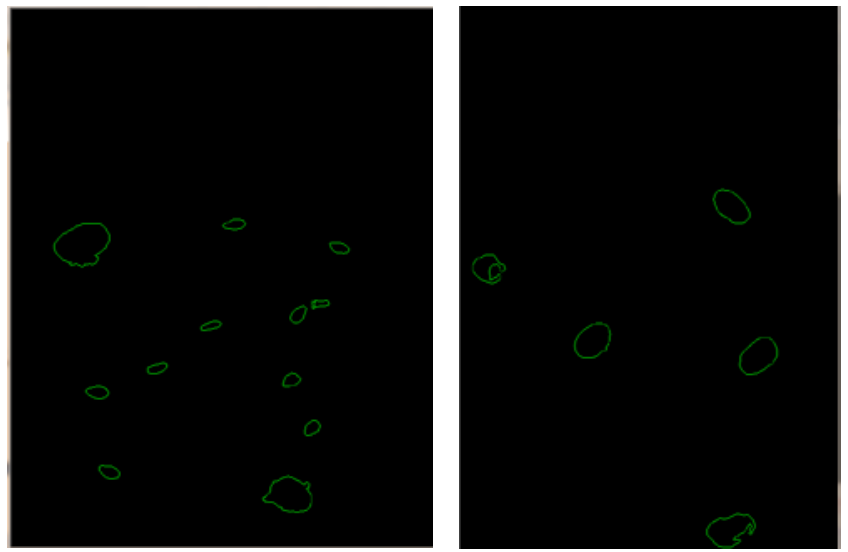

Figure.6 Contour images of rice impurity

The above experimental results show that the proposed method not only could accurately detect impurity but also accurately mark the location information of rice impurity.

\section{Conclusions}

To solve the existing methods fail to meet the requirements of rapid and precise inspection, the paper presents a rice impurity vision inspection method based OpenCV. The method mainly included four parts: Gray Processing, image binarization and blob detection. First, gray processing could significantly enhance impurity' information and achieve the goal in both sketching out approximately contours and showing details of impurity. Secondly the paper adopted OTSU method to effectively extract impurity and then improve accuracy of vision inspection; finally, through the Blob algorithm the paper quickly and accurately extract connected regions and calculated out its area and perimeter parameters for locating. In addition, this paper focused more on to propose the practical and easy to implement the rice impurity vision inspection method. The next work of the paper would discussion and research fast classification theory\& methodology of rice quality vision inspection. 


\section{Acknowledgements}

This work was supported by Supported by Program for Innovative Research Team (in Science and Technology) in University of Henan Province under Grant 17IRTSTHN011, the Youth support program of Henan University of Technology under Grant 2016QNJH29 and the Doctoral Foundation of Henan University of Technology under Grant 2012BS062.

\section{References}

[1] Liang Ming, Meng Dawei. Quality Detection Method Research and Simulation Based on Rice Images [J].Computer Simulation,2012,29( 6):244-247.

[2] Cui Wenwen, Sun Yonghai, Wang Lu, et al. Rice Processing Level Detection Method Based on Image Texture Analysis [J]. Journal of the Chinese Cereals and Oils Association, 2016, 31(6):146-149.

[3] Guo Wenchuan, Zhu XinHua, Wang Lu, et al. Research Progress of Machine Vision Technology in Grains Identification and classification [J]. Cereal \&Feed Industry, 2016, 31(6):146-149.

[4] Xu Li, Qian Minjuan, Fang Ruming, et al. Image Process Technique to Cognize the External Qualities and Milling Degree of Rice [J]. Transactions of the CSAE, 1996, 12(3):172-175.

[5] Liu W, Tao Y, Siebenmorgen T.J, et al. Digital image analysis method for rapid measurement of rice degree of milling [J]. Cereal Chemistry, 1998, 75(3): 380-385.

[6] Yadav B.K, Jindal V.K. Monitoring milling quality of rice by image analysis [J]. Computers and Electronics in Agriculture, 2001, 33(1):19-33.

[7] Wan Y N, Lin C M, Chiou J. F. Adaptive classification method for an automatic grain quality inspection system using machine vision and neural network[C]. 2000 ASAE Annual International Meeting, Milwaukee, pp.1-19.

[8] Rahul B, Ajay P.S.C. An Efficient Method for Quality Analysis of Rice Using Machine Vision System [J]. Journal of Advances in Information Technology,2015,6(3):140-145.

[9] Lurstwut B, Pornpanomchai C. Application of Image Processing and Computer Vision on Rice Seed Germination Analysis[J]. International Journal of Applied Engineering Research, 2016, 11(9): 6800-6807.

[10] Robert L. OpenCV 2 Computer Vision Application Programming Cookbook [M]. Birmingham, UK: Packt Publishing Ltd, 2011.

[11] Alasdair McAndrew. A Computational Introduction to Digital Image Processing, Second Edition [M]. Florida, USA: CRC Press, 2015

[12]He Zhiyong, Sun Lining, Chen Liguo. Fast computation of threshold based on Otsu Criterion [J]. Acta Electronica Sinca, 2013,41(2):267-272

[13] Zheng Chengyong, L i Hong. Vehicle license plate characters segmentation method using character's entirety and blob analysis [J]. J. Huazhong Univ. of Sci. \& Tech. (Natural Science Edition), 2010,38(3):88-91 